Annales Geophysicae (2003) 21: 661-669 (c) European Geosciences Union 2003

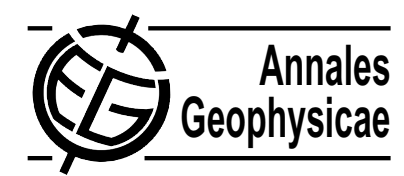

\title{
A note on the ring current in Saturn's magnetosphere: Comparison of magnetic data obtained during the Pioneer-11 and Voyager-1 and -2 fly-bys
}

\author{
E. J. Bunce and S. W. H. Cowley \\ Department of Physics and Astronomy, University of Leicester, Leicester LE1 7RH, UK
}

Received: 21 August 2002 - Revised: 26 November 2002 - Accepted: 6 December 2002

\begin{abstract}
We examine the residual (measured minus internal) magnetic field vectors observed in Saturn's magnetosphere during the Pioneer-11 fly-by in 1979, and compare them with those observed during the Voyager-1 and -2 flybys in 1980 and 1981 . We show for the first time that a ring current system was present within the magnetosphere during the Pioneer-11 encounter, which was qualitatively similar to those present during the Voyager fly-bys. The analysis also shows, however, that the ring current was located closer to the planet during the Pioneer-11 encounter than during the comparable Voyager-1 fly-by, reflecting the more compressed nature of the magnetosphere at the time. The residual field vectors have been fit using an adaptation of the current system proposed for Jupiter by Connerney et al. (1981a). A model that provides a reasonably good fit to the Pioneer-11 Saturn data extends radially between 6.5 and $12.5 R_{S}$ (compared with a noon-sector magnetopause distance of $17 R_{S}$ ), has a north-south extent of $4 R_{S}$, and carries a total current of 9.6 MA. A corresponding model that provides a qualitatively similar fit to the Voyager data, determined previously by Connerney et al. (1983), extends radially between 8 and $15.5 R_{S}$ (compared with a noon-sector magnetopause distance for Voyager-1 of $23-24 R_{S}$ ), has a north-south extent of $6 R_{S}$, and carries a total current of 11.5 MA.
\end{abstract}

Key words. Magnetospheric physics (current systems, magnetospheric configuration and dynamics, planetary magnetospheres)

\section{Introduction}

The magnetic environment of Saturn has been investigated to date by only three spacecraft, by Pioneer-11, and Voyagers1 and -2 , which undertook fly-bys of the planet in 1979, 1980, and 1981, respectively. All three spacecraft entered the planet's magnetosphere in the vicinity of the noon meridian,

Correspondence to: E. J. Bunce

(emma.bunce@ion.le.ac.uk) and exited on the dawn side, with Pioneer-11 and Voyager-2 exiting nearly along the dawn meridian, while Voyager-1 exited further down the tail (e.g. Smith et al., 1980a; Ness et al., 1981, 1982). One of the main features of the magnetic field in the central parts of the magnetosphere observed in Voyager data was the signature of a substantial "ring current" carried by charged particles of the magnetospheric plasma. The existence of this current was first recognised from depressions in the strength of the field below that expected for the internal field of the planet alone (Ness et al., 1981, 1982), and was subsequently modelled in some detail by Connerney et al. (1981b, 1983). The ring current inferred from the Voyager data is located in the radial range $\sim 8-16 R_{S}$ in a region which is a few $R_{S}$ wide on either side of the equatorial plane, and carries a total azimuthal current of $\sim 10 \mathrm{MA}$. This region thus corresponds roughly to the "extended plasma sheet" plasma region of Sittler et al. (1983) (see also, e.g. Richardson 1986, 1995). The peak magnetic perturbations in the poloidal field components due to the current are $\sim 10 \mathrm{nT}$ in magnitude, and are thus comparable with the magnitude of the internal planetary field at the equator at $\sim 10 R_{S}$, which is $\sim 20 \mathrm{nT}$.

In analyses published to date, however, it has never been clear whether similar magnetic perturbations were also observed during the Pioneer-11 fly-by. Indications that a ring current was indeed present was already inferred by Smith et al. (1980b) from depressions in the strength of the magnetospheric field, similar to those observed in Voyager data by Ness et al. (1981, 1982). In addition, spherical harmonic analysis of the field in the inner region (within $\sim 8 R_{S}$ ) consistently yields the presence of a quasi-uniform "external" field in all three fly-by data sets (Davis and Smith, 1990; see also Connerney et al., 1984). This field is of magnitude $\sim 10 \mathrm{nT}$ and is directed predominantly northward, as would be the case for an eastward-flowing equatorial ring current located at larger distances. These results provide initial evidence for the presence of a ring current during the Pioneer11 encounter, which is similar to that observed during the Voyager fly-bys. However, the nature of the Pioneer-11 perturbation fields has never been determined in detail, nor has 
the distribution of current been estimated. With the arrival of the Cassini spacecraft at Saturn now imminent (in July 2004), here we therefore undertake a brief analysis of the Pioneer-11 magnetic field data, comparing results with those obtained earlier from Voyager magnetometer data. Corresponding analysis of data from all three fly-bys provides the only means at present of estimating the range of conditions likely to be encountered by Cassini. We show for the first time that magnetic residual fields were indeed present during the Pioneer-11 encounter, which was of similar form to those observed during the Voyager fly-bys. We also make an estimate of the associated distribution and strength of the magnetospheric ring current from the Pioneer-11 data.

\section{Magnetic models}

Analysis of the fly-by magnetic data presented here consists of two steps. First, we use a model of Saturn's internal magnetic field to subtract the planetary field from the measured values. This yields the residual field vectors which are due to other current systems, principally the ring current field at middle and inner distances. Second, the field of a suitablyparameterised ring current model is then compared with the residual vectors, and the parameters varied until an adequate match is achieved. The magnetic models used to achieve these steps will be briefly described here.

For the internal planetary field we employ the SPV (Saturn Pioneer Voyager) model, based on an analysis of the near-planet data from all three spacecraft (Davis and Smith, 1990). This model is axially symmetric about the planet's spin axis, and employs three terms, namely the axial dipole, quadrupole, and octupole terms. The corresponding coefficients are $g_{1}^{0}=21160, g_{2}^{0}=1560$, and $g_{3}^{0}=2320 \mathrm{nT}$, for a Saturn radius of $60330 \mathrm{~km}$. We should comment, however, that none of our conclusions would be substantially altered if the other commonly-employed internal field model had been used, namely the equally axially-symmetric $Z_{3}$ model of Connerney et al. (1982) (which has instead $g_{1}^{0}=21184$, $g_{2}^{0}=1606$, and $g_{3}^{0}=2669 \mathrm{nT}$ for the same Saturn radius).

The model of the ring current used here is the same as that employed previously by Connerney et al. (1981b, 1983), for ease of comparison with previous results. The basic current element in this model is an axially-symmetric disc of current of constant half-thickness $D$, which has an inner edge at a cylindrical radial distance $R$, and which then extends radially to infinity. The azimuthal current within the disc varies inversely with the radial distance from the axis. A current disc of finite radial extent is then simulated by adding another similar disc of larger inner radius, whose current intensity is the same as that of the first, but which flows in the opposite sense. The model current density distribution is thus given by

$$
\boldsymbol{j}(\rho)=\frac{I_{0}}{\rho} \hat{\varphi}
$$

for $R_{1} \leq \rho \leq R_{2}$ and $-D \leq z \leq D$, and is zero otherwise. This model was first developed to describe the azimuthal current sheet in Jupiter's magnetosphere (Connerney et al., 1981a), and was later adapted with modified coefficients for Saturn. The Saturn coefficients obtained by Connerney et al. (1983) from fits to the Voyager data were $R_{1}=8 R_{S}$, $R_{2}=15.5 R_{S}, D=3 R_{S}$, and $\mu_{0} I_{0}=60.4 \mathrm{nT}$. It should be noted that this current system has an essentially "square" cross section ( $7.5 R_{S}$ in $\rho$ by $6 R_{S}$ in $\left.z\right)$, compared with the disc-like system at Jupiter (for which instead $R_{1}=5 R_{J}$, $R_{2}=50 R_{J}$, and $D=2.5 R_{J}$ ).

The exact solution for the field components of this current model is given by Fourier-like integrals over Bessel functions which must be evaluated numerically. The initial results for the fits to the observed planetary current sheet fields at Jupiter and Saturn were both obtained using this formulation (Connerney et al., 1981a, b, 1983). However, in the original paper describing the current model, Connerney et al. (1981a) also provided some analytic approximate forms, which were corrected by Acuña et al. (1983), and later systematised (such that, for example, the approximate forms are exactly divergence-free) by Edwards et al. (2001). Subsequent application of the model to the jovian system by other authors (see e.g. the references cited in Edwards et al., 2001) has then generally employed these analytic approximations. Comparison shows that these analytic forms provide very accurate approximations away from the inner and outer edges of the current disc (e.g. Acuña et al., 1983; Edwards et al., 2001). However, at the inner and outer edges the approximate cylindrical radial component undergoes a discontinuity, and the axial component, while continuous, is reduced in magnitude compared with the full solution obtained from the numerical integrals. In the jovian environment these limitations are of little consequence, because at the inner edge of the current sheet at $5 R_{J}$ the current sheet field magnitudes ( $\sim 100 \mathrm{nT}$ in the radial component and $\sim 200 \mathrm{nT}$ in the axial), are small compared with the internal planetary field of $\sim 3500 \mathrm{nT}$. The analytic forms then provide very accurate approximations at significantly larger distances where the current sheet provides the dominant field (beyond $\sim 15 R_{J}$ ), out to the outer limit of validity of the model at $\sim 30 R_{J}$. However, for the "squarer" Saturn current model, essentially any point which lies in the vicinity of the current distribution lies "close to the edges" in the above sense. In this case the above-cited approximate forms do not work well.

This conclusion is illustrated in Fig. 1, which shows a comparison between numerically integrated field values and those obtained from the analytic approximations, specifically for the Connerney et al. (1983) Saturn ring current parameters given above. Figure 1a shows the cylindrical radial component of the ring current field $B_{R C \rho}$ at $z=3 R_{S}$ (the northern edge of the model ring current where the radial field maximises), plotted versus cylindrical radial distance from the axis $\rho$ over the range $0 \leq \rho \leq 20 R_{S}$. The solid line shows $B_{R C \rho}$ obtained numerically from the exact integral formulas of Connerney et al. (1981a) (see also Edwards et al., 2001), while the dashed lines show the analytic approxi- 

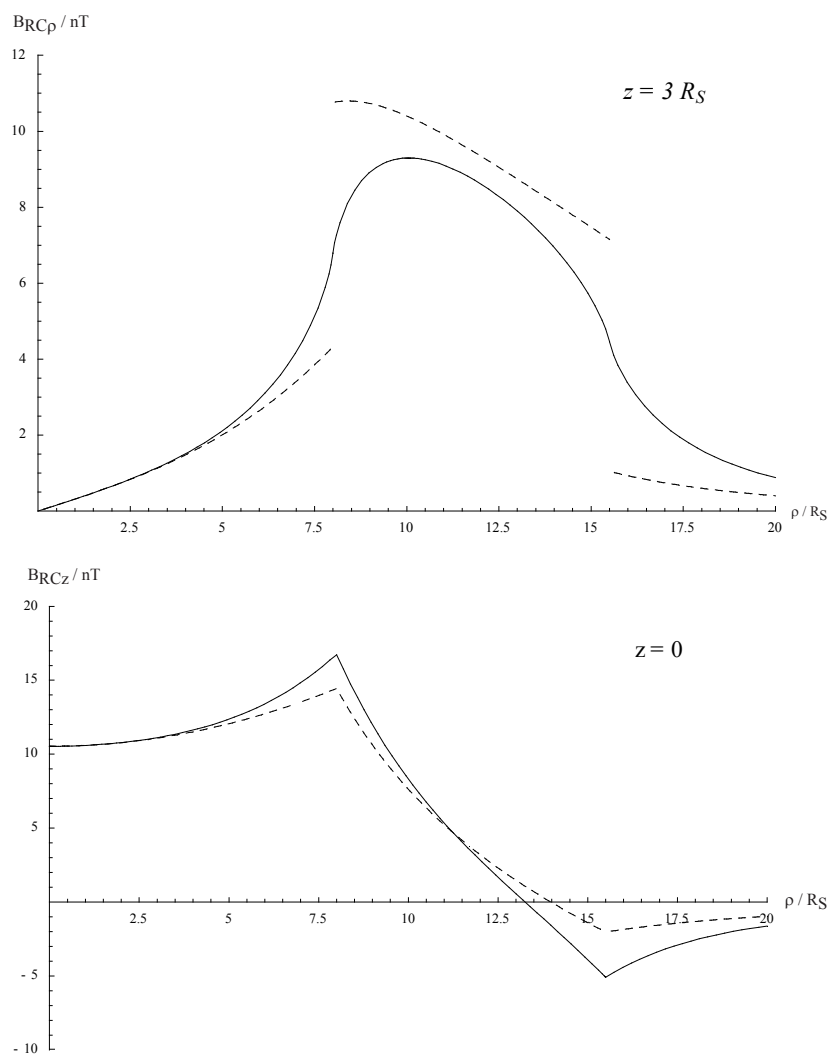

Fig. 1. Plots of the field components produced by the ring current in Saturn's magnetosphere, as determined from fits to Voyager-1 and -2 magnetic data by Connerney et al. (1983). The solid lines show values determined from numerical evaluation of exact integral expressions (Connerney et al., 1981a), while the dashed lines show analytic approximations, specifically the improved forms given by Edwards et al. (2001). Plot (a) shows the cylindrical radial component of the ring current field $B_{R C \rho}$ at $z=3 R_{S}$ (the northern edge of the ring current) versus cylindrical radial distance $\rho$. Plot (b) similarly shows the axial component of the ring current field $B_{R C} z$ at $z=0$, also plotted versus $\rho$ over the same range.

mations, derived specifically from the improved expressions given by Edwards et al. (2001). The approximations are defined and plotted in three ranges of $\rho$, corresponding to cylindrical radial distances which are less than, within, and greater than the range occupied by the current. It can be seen that major discontinuities occur in the approximate fields at the inner and outer edges of the current sheet at $\rho$ values of 8 and $15.5 R_{S}$, respectively. Figure $1 \mathrm{~b}$ similarly shows the ring current axial field $B_{R S \rho}$ at $z=0$ (where this component also maximises), also plotted versus $\rho$ over the same range. In this case the approximate values are continuous at the radial boundaries, but are somewhat less than the full numerical values. Overall, it can be seen that these analytic forms do not provide accurate approximations for Saturn's ring current field, except at small radial distances near the planet.

In this paper, therefore, we have used model field values obtained numerically from the exact integral formulas to compare with the spacecraft fly-by data as in the original work of Connerney et al. (1981a, 1983). However, we note that subsequent to this work being completed, Giampieri and Dougherty (2002) have derived exact solutions for the field, as an infinite series, the leading terms of which are the approximate forms given by Edwards et al. (2001). In this case, while discontinuities still exist when switching between approximations which are appropriate to the various regimes, as above, their magnitude can be made arbitrarily small by including successively higher order terms in the series. Use of the first six terms has been employed in modelling reported by the above authors, resulting in the "steps" being reduced to modest size. Here, however, we employ numerical integration of the exact integral formulae, as just indicated, such that no "steps" occur at all.

\section{Results}

We begin our presentation of results by showing data for the two Voyager passes discussed previously by Connerney et al. (1983), for comparison with the Pioneer-11 data which follows. Figure 2 shows the trajectories of the spacecraft plotted in cylindrical $(\rho, z)$ coordinates, while Figs. 3 and 4 show plots of the residual field components and fitted model ring current fields for Voyager-1 and -2 , respectively. The dashed rectangle in Fig. 2 also shows the ring current region modelled by Connerney et al. (1983), as given above. It can be seen that Voyager-1 inbound passed radially through the model current south of the equatorial plane, while outbound it passed more obliquely through the current region at northern latitudes. Voyager-2, on the other hand, did not pass through the model current region at all, but instead remained north of the region inbound, and south of the region outbound. We recall from the Introduction that the local time of the inbound passes was near noon $(\sim 13: 00 \mathrm{LT})$ for both Voyagers, while the outbound passes were in the postmidnight sector ( 03:00 LT) for Voyager-1 and near dawn ( 06:00 LT) for Voyager-2.

Figures 3 and 4 show two (Earth) days of data each, spanning the interval from the inbound magnetopause encounters (leftmost dashed line(s) marked "MP"), through closest approach to the planet (central dashed line marked "CA"), to radial distances of $\sim 20 R_{S}$ outbound. We have deleted some spurious field values near to closest approach in both plots which were affected by insufficiently precise attitude reconstruction during spacecraft manoeuvres (see Connerney et al., 1983). The plots do not encompass the outbound magnetopause crossings, which in both cases were multiple and occurred at (spherical) radial distances $43-47 R_{S}$ for Voyager-1 and 50-70 $R_{S}$ for Voyager-2 (e.g. Behannon et al., 1983). The top three panels of each figure show the residual field components (measured value minus SPV internal model) versus time in cylindrical coordinates referenced to the planet's spin (and magnetic) axis. The dotted curve in the upper two panels also shows the $\rho$ and $z$ field components of the current system modelled by Connerney et al. (1983), the parameters of which were indicated in rela- 


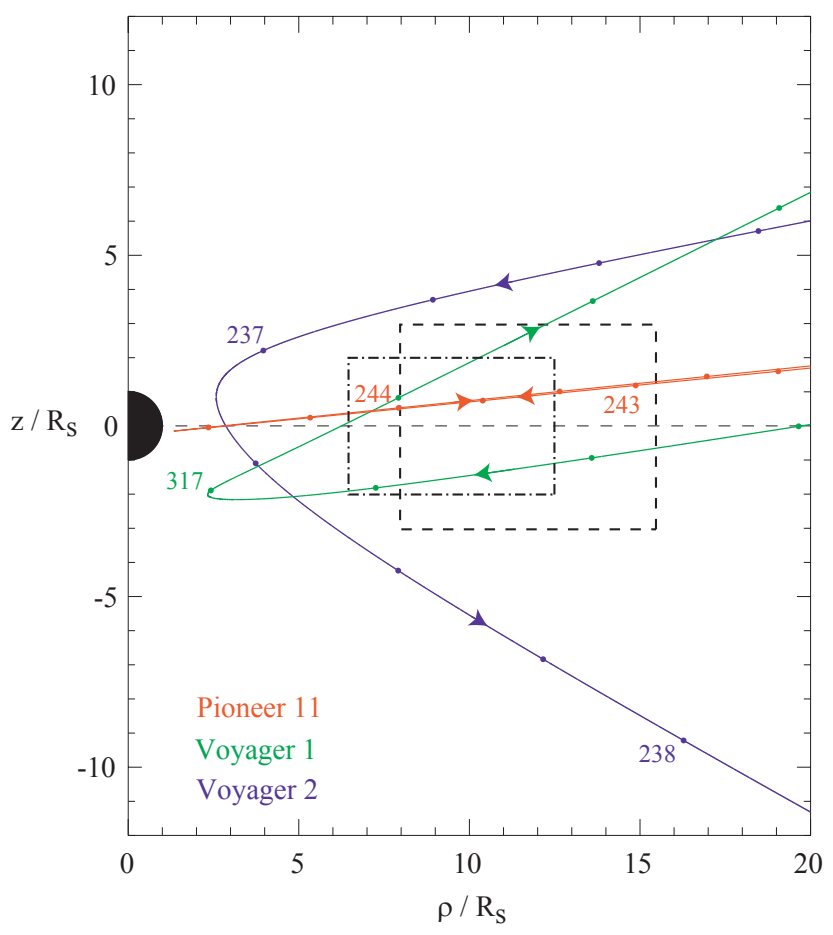

Fig. 2. Plot showing the trajectories of Pioneer-11, and Voyager-1 and -2 relative to Saturn in cylindrical coordinates. The horizontal axis shows the perpendicular distance from the planet's spin (and magnetic axis), $\rho$, while the vertical axis shows the distance along this axis from the equatorial plane, $z$. These distances are given in units of Saturn's radius, $R_{S}$, taken to be $60330 \mathrm{~km}$. The tic-marks on the trajectories indicate 6-h intervals, while the arrows show the direction of motion of the spacecraft, which was essentially along the same path inbound as outbound for Pioneer-11. The dashed rectangle marks the ring current region modelled by Connerney et al. (1983) based on Voyager field data, which lies between 8 and $15.5 R_{S}$ in $\rho$, and between -3 and $+3 R_{S}$ in $z$. The dot-dashed rectangle marks the ring current region modelled here using Pioneer-11 field data, which lies between 6.5 and $12.5 R_{S}$ in $\rho$, and between -2 and $+2 R_{S}$ in $z$.

tion to Eq. (1) above. The azimuthal component in the third panel is shown for completeness, noting that since the SPV model is axi-symmetric and hence has zero azimuthal component, the plotted value represents the total azimuthal field measured. In addition, since the ring current model is also axi-symmetric, the model's azimuthal field is also zero. We note that Connerney et al. (1983) have modelled the principal azimuthal field signature observed near the closest approach, together with apparently related variations in the radial and axial fields, as being due to a field-aligned current which flows into the nightside dusk ionosphere at $\sim 80^{\circ}$ southern latitude. The lower two panels of the figures show the location of the spacecraft in cylindrical $(\rho, z)$ coordinates. The vertical dotted lines show the intervals during which the spacecrafts were within the cylindrical radial range of the model ring current, i.e. with $\rho$ values between 8 and $15.5 R_{S}$ (marked "RC" in the figures).

Considering first the Voyager-1 pass shown in Fig. 3, it can be seen that the magnetosphere was relatively expanded during this encounter, due to low solar wind dynamic pressure, with (five) inbound magnetopause crossings occurring at (spherical) radial distances between 23 and $24 R_{S}$. Relatively steady conditions during the encounter have been inferred by Behannon et al. (1983) from the commensurate boundary locations observed outbound. The outer edge of the inbound ring current can be recognised in the negative minimum in the $B_{z}$ field component occurring at $\sim 10: 00$ UT on day 317, as reproduced by the model (compare with Fig. 1b). Following the minimum, $B_{z}$ increased near-linearly with time before plateauing at a positive value somewhat lower than that of the model after $\sim 17: 00 \mathrm{UT}$, associated with the ring current inner edge. The radial component $B_{\rho}$ was relatively weak and negative during this interval, associated with the modest spacecraft displacement south of the equator. Subsequently, during the outbound pass, residual fields were observed which were similar to those inbound, though the $B_{z}$ field was modestly stronger and in better general agreement with the model. The $B_{\rho}$ component reversed in sense after the spacecraft crossed the equator, and then attained higher values than inbound, due principally to the larger distance of the spacecraft from the equatorial plane (see Fig. 2). Overall, and with the exception of the region near closest approach, the fields of the model current system can be seen to give a good general account of the observed residual field data.

The Voyager-2 data is shown in the same format in Fig. 4. In this case the magnetosphere was relatively compressed on entry, with a single magnetopause crossing at a (spherical) radial distance of $19 R_{S}$. A major expansion then took place some time during the encounter, though exactly when is not clear, leading to dawn meridian magnetopause crossings outbound at (spherical) distances between 50 and $70 R_{S}$, as noted above. Behannon et al. (1983) give reasons to suppose that the expansion was due to Saturn becoming immersed in the low dynamic pressure environment of Jupiter's magnetic tail, or filament thereof, during the encounter. The residual magnetic fields observed by Voyager-2 shown in Fig. 4 are of similar form to those observed by Voyager- 1 when the differing spacecraft trajectory is taken into account, with excellent agreement between measured and model values being found on the dawn outbound pass. On the inbound pass, however, the agreement is less satisfactory, with the residual fields again being rather smaller than those modelled, and with a differing spatial distribution. In particular we note that the reversal in the sense of the $B_{z}$ residual field from negative to positive occurred at a cylindrical radial distance of $\sim 11 R_{S}$ rather than at $\sim 15 R_{S}$, as modelled, with this component finally plateauing at $\sim 5.5 R_{S}$, as opposed to $8 R_{S}$ in the model. These relative positions are indicative of the presence of a weaker current system during the inbound pass which was displaced inwards from that modelled, the displacement probably being associated with the relative compression of the magnetosphere.

We now turn to the central results of this note, namely an examination of the residual fields observed by Pioneer-11. Two (Earth) days of data are again shown in Fig. 5, in the 

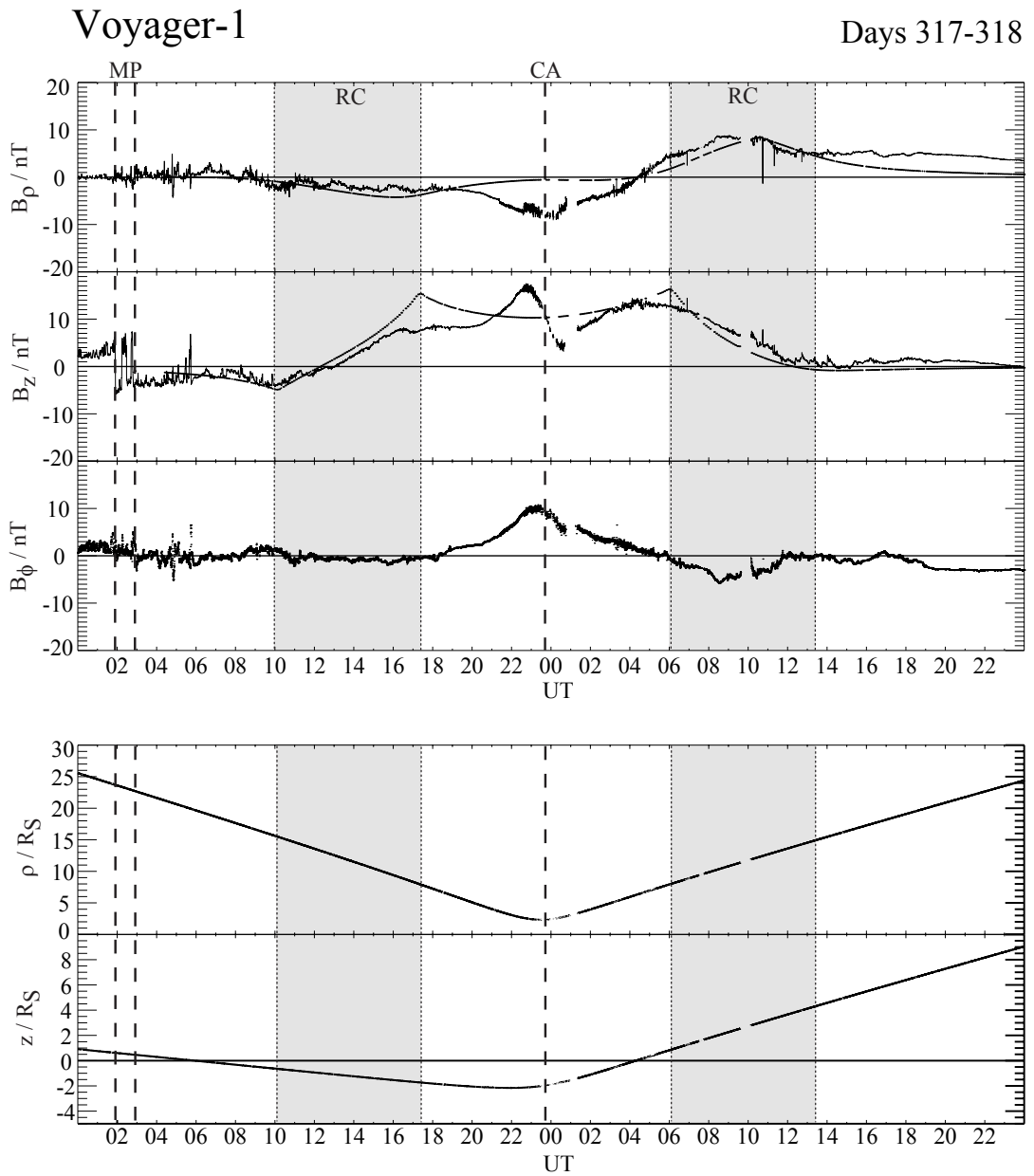

Fig. 3. Plot showing magnetic field and position data for the Voyager-1 fly-by of Saturn. Data for two (Earth) days are shown, corresponding to days 317 and 318 of 1980 . The top three panels show the residual field components in cylindrical coordinates referenced to the planet's spin (and magnetic) axis, namely from the top downwards, $B_{\rho}, B_{z}$, and $B_{\varphi}$ (in $\mathrm{nT}$ ). These residuals are the measured field minus the SPV model of the internal planetary field (which is zero in the case of the azimuthal component). The dotted lines show the components of the ring current field model derived by Connerney et al. (1983) (which again has zero azimuthal component). The model parameters (see Eq. (1)) are $R_{1}=8 R_{S}, R_{2}=15.5 R_{S}, D=3 R_{S}$, and $\mu_{0} I_{0} \approx 60.4 \mathrm{nT}$. The lower two panels show the position of the spacecraft, namely the cylindrical radial distance from the planet's spin (and magnetic axis), $\rho$, and the distance along this axis from the equatorial plane, $z$. The left-hand vertical dashed lines marked "MP" show the positions of the first and last of five inbound magnetopause transitions (at spherical radial distances between 23 and $24 R_{S}$ ), while the central vertical dashed line marked "CA" shows the closest approach of the spacecraft to the planet (at a spherical radial distance of $3.1 R_{S}$ ). The vertical dotted lines indicate the intervals during which the spacecraft lay within the cylindrical radial range of the current sheet, that is within $8 \leq \rho \leq 15.5 R_{S}$ (marked "RC").

same format as Figs. 3 and 4 (the derivation of the model values will be discussed further below). Field vectors which are clearly spurious have again been removed from the plot, and we also note the presence of an extended data-gap following closest approach, which occurred during occultation of the spacecraft by the planet (Smith et al., 1980b). The spacecraft trajectory is also shown in cylindrical coordinates in Fig. 2, from which it can be seen that similar radial cuts were made through the expected current-carrying region both inbound and outbound, at modest displacements north of the equator. The spacecraft local time was $\sim 12: 00 \mathrm{LT}$ inbound, and $\sim 06: 00$ LT outbound. In this case the magnetosphere was even more compressed on entry than during the Voyager-2 fly-by, with one magnetopause crossing occurring inbound at a spherical radial distance of $17 R_{S}$. The compression was due to the arrival of a fast solar wind stream just prior to the fly-by (Smith et al., 1980b), with relaxation occurring some time during the encounter, judging from the outbound boundary positions (with magnetopause encounters occurring between 30 and $40 R_{S}$ on the dawn meridian).

Examination of the magnetic data shows immediately that similar residual fields were observed by Pioneer-11 as by Voyagers- 1 and -2 , indicative of the presence of a ring current system during the encounter interval. In fact, the overall profile for Pioneer-11 is very similar to that for Voyager1 , reflecting the similar nature of the encounter trajectories. Specifically, we note the negative minimum in $B_{z}$ at $\sim 03: 00$ UT on day 244, marking the outer boundary of the 


\section{Voyager-2}

Days 237-238
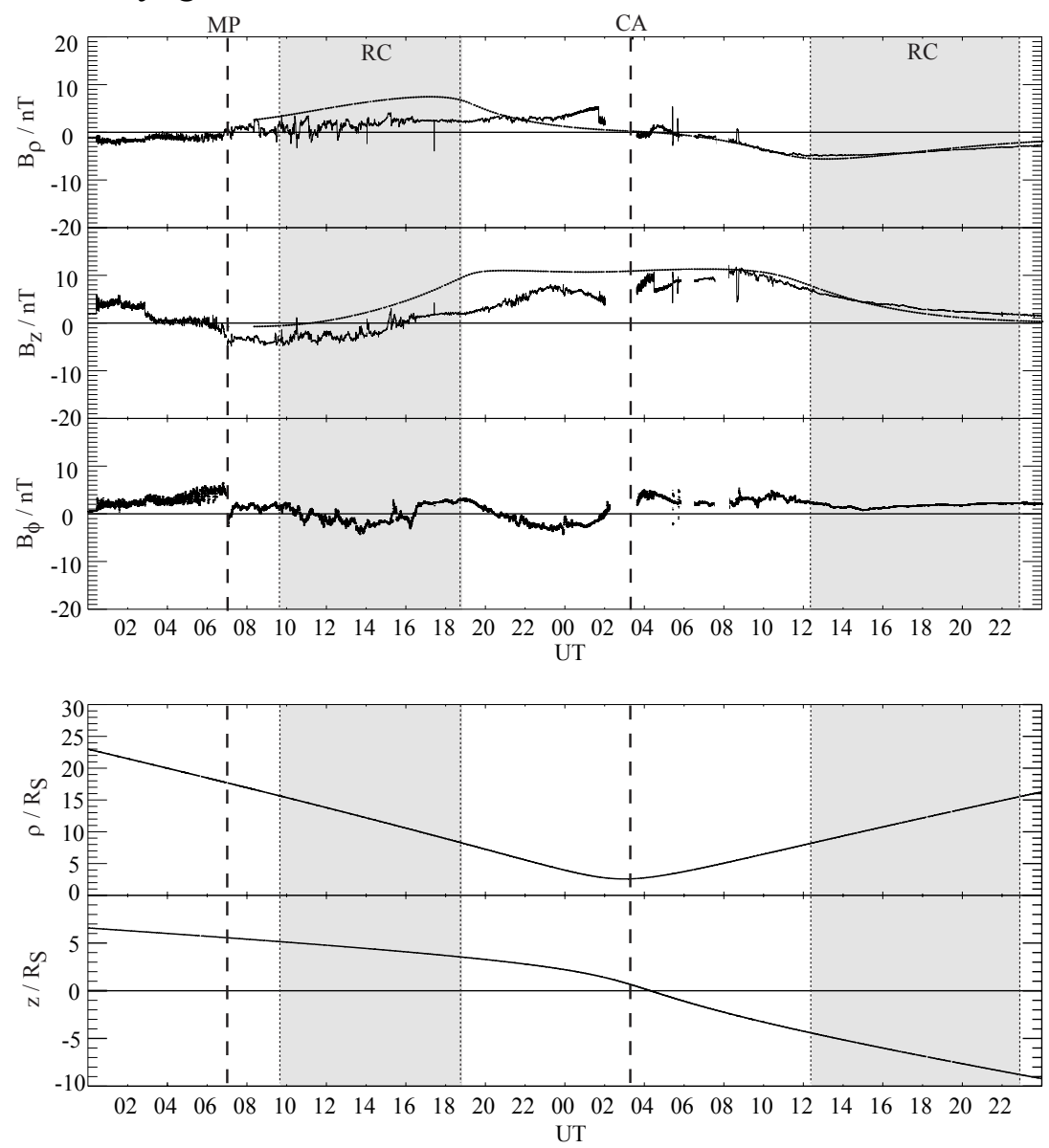

Fig. 4. Plot showing magnetic field and position data for the Voyager-2 fly-by of Saturn, in the same format as Fig. 3. Data for two (Earth) days are again shown, corresponding to days 237 and 238 of 1981. The fitted ring current model is again that derived by Connerney et al. (1983). In this case only one magnetopause crossing took place inbound at a spherical radial distance of $19 R_{S}$, and the closest approach occurred at a spherical radial distance of $2.7 R_{S}$.

dayside ring current, the near-linear rise in $B_{z}$ that follows, and the plateauing before the closest approach (though now with significant fluctuations in the field) that marks the inner edge. Following the closest approach, we also note somewhat larger positive residual $B_{z}$ fields than observed inbound, again as for both Voyager- 1 and -2 . These data thus serve to confirm the presence of a ring current during the Pioneer-11 fly-by, whose magnetic perturbations were qualitatively similar to those observed by Voyagers- 1 and -2 . The main qualitative difference between the residuals observed by these spacecraft is that in the case of Pioneer-11, positive radial components were observed both before and after closest approach, due to the spacecraft remaining north of the equatorial plane both inbound and outbound. We also note, however, the marked oscillatory behaviour in the Pioneer-11 radial component in the outer region and in the azimuthal component, recently discussed by Espinosa and Dougherty (2000), which remains of unknown origin.

Although these data thus clearly demonstrate the presence of ring current effects in Pioneer-11 data, it is also clear from
Fig. 5 that the spatial distribution of the current was rather different during the Pioneer-11 fly-by than for Voyager-1, to which it is most qualitatively similar. This is shown explicitly in Fig. 6, where we plot the residual $B_{z}$ field versus cylindrical radial distance $\rho$ for both Pioneer-11 (red) and Voyager-1 (green) over the range of distances between 4 and $24 R_{S}$ (the dotted lines show model fields that will be discussed below). The upper and lower panels show the fields on the inbound and outbound passes of both spacecraft, respectively, which occurred in similar local time sectors, as indicated above. Inbound magnetopause crossings are marked in the upper panel. It can be seen that very similar profiles were observed by the two spacecraft on both passes, but with corresponding features occurring at smaller radial distances on the Pioneer-11 pass than on the Voyager-1 pass. Specifically, on the inbound passes near noon, the negative minimum in the residual $B_{z}$ field marking the outer edge of the ring current occurred at a (cylindrical) radial distance of $\sim 12-13 R_{S}$ for Pioneer-11, compared with $\sim 15-16 R_{S}$ for Voyager-1, while the outer edge of the inbound $B_{z}$ "plateau", 
Pioneer-11
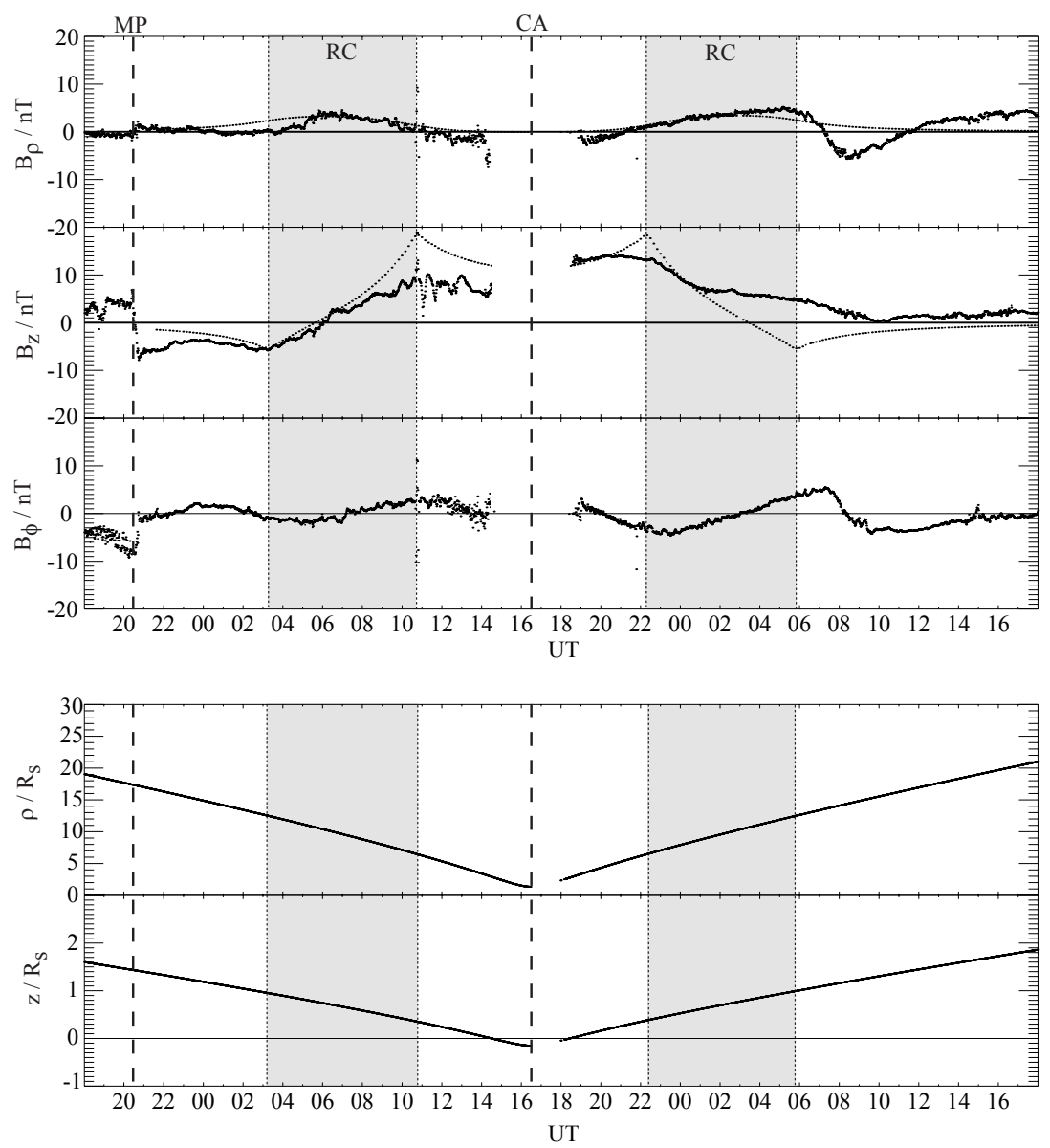

Fig. 5. Plot showing magnetic field and position data for the Pioneer-11 fly-by of Saturn, in the same format as Fig. 3. Data for two (Earth) days are again shown, corresponding to the interval from 18:00 UT on day 243 to 18:00 UT on day 245 of 1979. The fitted ring current model has parameters $R_{1}=6.5 R_{S}, R_{2}=12.5 R_{S}, D=2 R_{S}$, and $\mu_{0} I_{0} \approx 76.5 \mathrm{nT}$, as discussed in the text. Only one magnetopause crossing took place inbound at a spherical radial distance of $17 R_{S}$, and the closest approach occurred at a spherical radial distance of $1.35 R_{S}$.

marking the inner edge of the ring current, occurred at $\sim 6-$ $7 R_{S}$ for Pioneer-11, compared with $\sim 8-9 R_{S}$ for Voyager-1. These values are indicative of a current system which was displaced towards smaller distances from the planet during the Pioneer-11 encounter compared with the Voyager-1 flyby. This no doubt reflects the more compressed nature of the magnetosphere during the Pioneer-11 fly-by (as also inferred qualitatively above from the Voyager-2 inbound data), with inbound magnetopause crossings occurring at $\sim 17 R_{S}$ for Pioneer-11 compared with $\sim 23-24 R_{S}$ for Voyager-1, as can be seen in the upper panel of the figure. Correspondingly, on the dawn-side outbound passes, the peak positive $B_{z}$ residual field occurred at a cylindrical radial distance of $\sim 4.5-5.5 R_{S}$ for Pioneer-11, but at $\sim 6-7 R_{S}$ for Voyager-1. We note, however, that the magnitude of the small- $\rho$ residual $B_{z}$ field was essentially the same on the two passes.

Due to the differing spatial distributions of current, the ring current model derived by Connerney et al. (1983) to describe the Voyager magnetic data is not appropriate to describe the Pioneer-11 data. In Fig. 6 the green dotted line shows the original Connerney et al. (1983) model field evaluated on the Voyager-1 trajectory, plotted for primary comparison with the Voyager-1 residual data shown. The purple dotted line then shows results for the same current model but evaluated on the Pioneer-11 trajectory. This is very similar to the green-dotted line for Voyager-1, due to the similarity of the trajectories of the spacecraft and the relative insensitivity of the model's $B_{z}$ field to modest north-south spatial displacements. While this model thus gives a good overall account of the Voyager-1 data (as already seen in Fig. 3 above), it can be seen that it does not provide a good description for Pioneer-11. In order to make a clear and simple comparison, we have therefore varied the current sheet parameters until a model has been obtained that fits the Pioneer-11 data in a manner which is qualitatively similar to the fit of the Connerney et al. (1983) model to the Voyager-1 data. This has been achieved in a straightforward and physical way by examining those features of the data that are the most revealing of and sensitive to the various parameters of the model. First, the values of $R_{1}$ and $R_{2}$ have been chosen by relating the ex- 


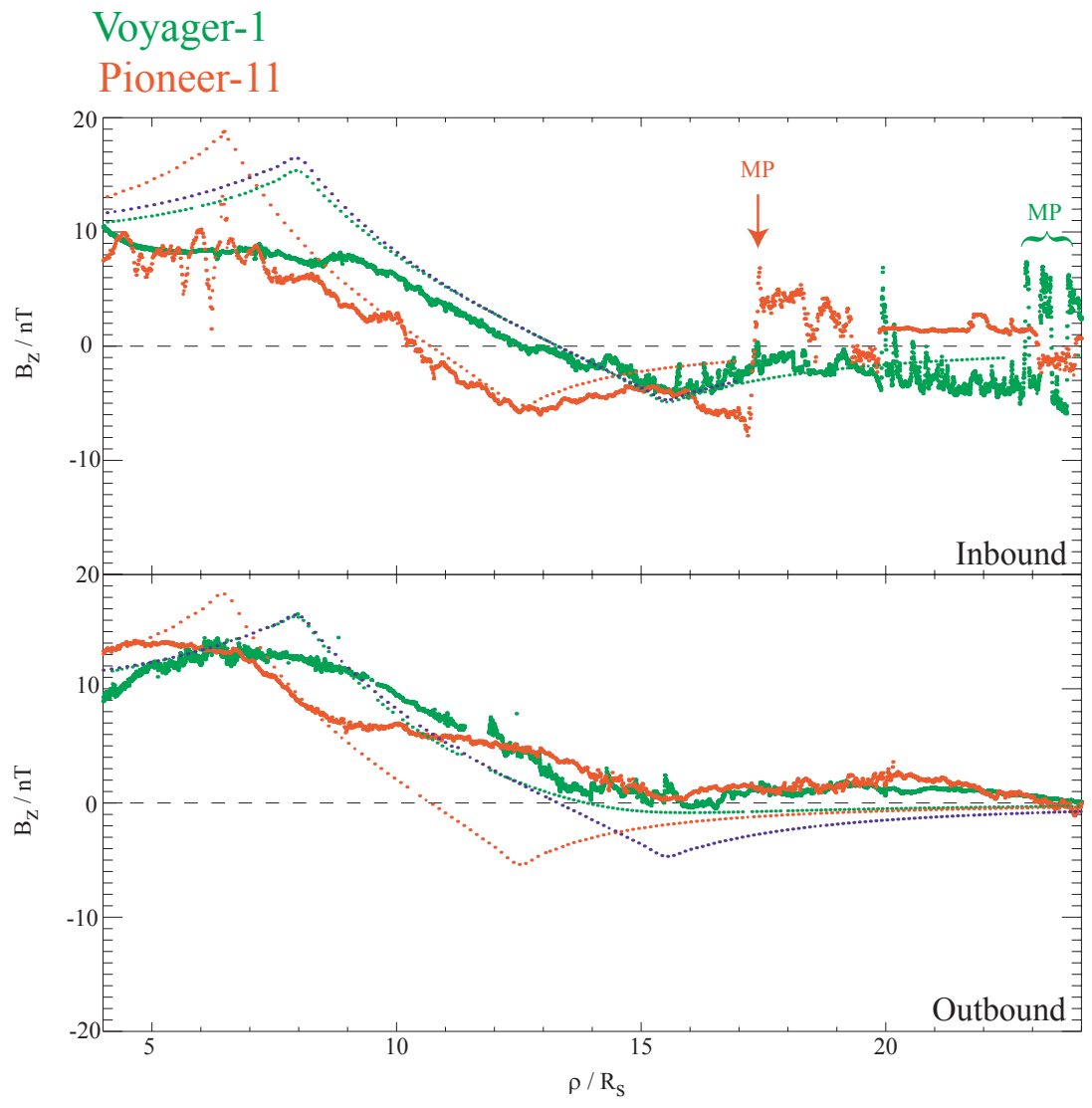

Fig. 6. Comparison of the residual $B_{z}$ fields observed by Pioneer-11 (red) and Voyager-1 (green), plotted versus cylindrical radial distance $\rho$ between 4 and $24 R_{S}$. The top panel shows the residuals observed on the inbound passes (at $\sim 12: 00$ and $\sim 13: 00$ LT for Pioneer-11 and Voyager-1, respectively), while the bottom panel shows those observed on the outbound passes (at $\sim$ 06:00 and $\sim 03: 00$ LT, respectively). Inbound magnetopause crossings are also marked in the upper panel (none occurred within the range on the outbound passes). The dotted lines also show the fields of the fitted model ring currents. The model field for Voyager-1 (green) is that derived by Connerney et al. (1983), as described in the text and caption to Fig. 3, while the model for Pioneer-11 (red) is that derived here, as described in the text and caption to Fig. 5. The purple dotted line shows the results for the Connerney et al. (1983) model derived for the Pioneer-11 trajectory, which does not provide a good fit.

trema in the model $B_{z}$ curve (see Fig. 1b) to the features of the observed $B_{z}$ profile. Second, noting that the model $B_{z}$ field at (and near) the origin is given by

$$
\begin{aligned}
B_{z 0}= & \frac{\mu_{0} I_{0}}{2}\left[\operatorname { l o g } \left[\left(\frac{\left(\left(R_{1} / D\right)^{2}+1\right)^{\sqrt{2}}+1}{\left(\left(R_{1} / D\right)^{2}+1\right)^{\sqrt{2}}-1}\right)\right.\right. \\
& \left.\left.\left(\frac{\left(\left(R_{2} / D\right)^{2}+1\right)^{\sqrt{2}}-1}{\left(\left(R_{2} / D\right)^{2}+1\right)^{\sqrt{2}}+1}\right)\right]\right]
\end{aligned}
$$

(see, e.g. Edwards et al., 2001), the desired amplitude of the ring current $B_{z}$ field near the closest approach, chosen to be $\sim 11$ nT for the Pioneer-11 data, defines a relationship between $\mu_{0} I_{0}$ and $D$. These two parameters are then iterated together to find the combination that gives the best fit to $B_{\rho}$ while holding $B_{z 0}$ constant. This is entirely physically appropriate since the profile of the $B_{\rho}$ field is sensitive to the distribution of the current with $z$, and hence, to $D$. The values of the parameters so determined are $R_{1} \approx 6.5 R_{S}, R_{2} \approx$ $12.5 R_{S}, D \approx 2 R_{S}$, and $\mu_{0} I_{0} \approx 76.5 \mathrm{nT}$. These parameters yield the model curves shown in Figs. 5 and 6 (the red dotted line in Fig. 6), which can thus be seen to fit the Pioneer-11 data in a manner similar to the Connerney et al. (1983) fit to the Voyager-1 data, shown in Fig. 3. The cross-sectional region in which the current flows in our Pioneer-11 model is shown by the dot-dashed lines in Fig. 2. Comparison with the corresponding parameters of the Connerney et al. (1983) model, for which $R_{1}=8 R_{S}, R_{2}=15.5 R_{S}, D=3 R_{S}$, and $\mu_{0} I_{0}=60.4 \mathrm{nT}$, as given above, then indicates that during the Pioneer-11 fly-by the ring current was displaced inwards by $\sim 1.5 R_{S}$ at the inner edge, compared with Voyager-1, by $\sim 3 R_{S}$ at the outer edge, and was narrower in north-south extent by $\sim 2 R_{S}$. In addition, the total current flowing in the Pioneer-11 model ring current, given by $2 I_{0} D \log \left(R_{2} / R_{1}\right)$, is estimated as $\sim 9.6 \mathrm{MA}$, compared with $\sim 11.5 \mathrm{MA}$ for the Connerney et al. (1983) model. From a qualitative examination of the sensitivity of the model fit, we estimate that the uncertainties in the model parameters are $\sim \pm 0.5 R_{S}$ in the $R$ values, $\sim \pm 0.25 R_{S}$ in $D$, and $\sim 10-20 \%$ in $\mu_{0} I_{0}$. The difference in the total current between the Pioneer-11 and Voyager 
values thus appears to be of somewhat marginal significance.

We finally note that in their subsequent related work, Giampieri and Dougherty (2002) have also considered the fitting of the Connerney et al. (1983) ring current model to the Pioneer-11 data, but have instead employed a straightforward least-squares fitting approach, rather than the more physically-motivated fit derived here. The values they obtain for the overall fit to the Pioneer-11 data are $R_{1} \approx 6.4 R_{S}$, $R_{2} \approx 13.9 R_{S}, D \approx 1.8 R_{S}$, and $\mu_{0} I_{0} \approx 50.8 \mathrm{nT}$, which thus provide additional measures of the uncertainties in the values quoted above.

\section{Summary}

In this note we have examined the residual (observed minus internal) magnetic field vectors observed in Saturn's magnetosphere during the Pioneer-11 fly-by in 1979, and have compared them with those observed during the Voyager-1 and -2 fly-bys in 1980 and 1981. The principal result of the study is that we have provided the first unequivocal evidence for the presence of a ring current system within the magnetosphere during the Pioneer-11 fly-by, and have shown it to be qualitatively similar to those present during the Voyager encounters. We have also shown, however, that the ring current was located somewhat closer to the planet during the Pioneer-11 fly-by than during the comparable Voyager-1 encounter, reflecting the more compressed nature of the magnetosphere at the time. The corresponding noon-sector near-equatorial magnetopause traversals were at a (spherical) radial distance of $17 R_{S}$ for Pioneer-11 and 23-24 $R_{S}$ for Voyager-1. A current system that provides a reasonably good fit to the Pioneer-11 data extends radially between 6.5 and $12.5 R_{S}$, has a north-south extent of $4 R_{S}$, and carries a total current of 9.6 MA. The outer edge of the ring current was thus located $\sim 4.5 R_{S}$ inside the magnetopause, if no boundary motions occurred during the $\sim 7 \mathrm{~h}$ spacecraft transit between the two. We also note that the inner edge of the ring current coincided approximately with inner edge of the high- $\beta$ plasma region determined during the inbound Pioneer-11 pass by Frank et al. (1980). By comparison, the corresponding model derived previously by Connerney et al. (1983) from the Voyager data extends radially between 8 and $15.5 R_{S}$ (the outer boundary thus being located $\sim 8 R_{S}$ from the observed magnetopause), has a north-south extent of $6 R_{S}$, and carries a total current of 11.5 MA.

Acknowledgements. We would like to thank Stéphane Espinosa for the provision of Pioneer-11 magnetic field data, Joe Mafi of the Planetary Data System, UCLA for supplying the Voyager-1 and -2 magnetic field data employed. We would also like to thank Douglas Robinson and Karen Veal, who piloted elements of the work reported here during undergraduate research projects in the Department of Physics \& Astronomy at the University of Leicester. EJB was supported during the course of this study by PPARC Grant PPA/G/O/1999/00181 and SWHC by PPARC Senior Fellowship PPA/N/S/2000/00197.

Topical Editor T. Pulkkinen thanks two referees for their help in evaluating this paper.

\section{References}

Acuña, M. H., Behannon, K. W., and Connerney, J. E. P.: Jupiter's magnetic field and magnetosphere, in: Physics of the Jovian Magnetosphere, (Ed) Dessler, A. J., p. 1, Cambridge Univ. Press, Cambridge, UK, 1983.

Behannon, K. W., Lepping, R. P., and Ness, N. F.: Structure and dynamics of Saturn's outer magnetosphere and boundary regions, J. Geophys. Res., 88, 8791, 1983.

Connerney, J. E. P., Acuña, M. H., and Ness, N. F.: Modeling the Jovian current sheet and inner magnetosphere, J. Geophys. Res., 86, 8370, 1981a.

Connerney, J. E. P., Acuña, M. H., and Ness, N. F.: Saturn's ring current and inner magnetosphere, Nature, 292, 724, 1981b.

Connerney, J. E. P., Ness, N. F., and Acuña, M. H.: Zonal harmonic model of Saturn's magnetic field from Voyager 1 and 2 observations, Nature, 298, 44, 1982.

Connerney, J. E. P., Acuña, M. H., and Ness, N. F.: Currents in Saturn's magnetosphere, J. Geophys. Res., 88, 8779, 1983.

Connerney, J. E. P., Acuña, M. H., and Ness, N. F.: The $Z_{3}$ model of Saturn's magnetic field and the Pioneer 11 vector helium magnetometer observations, J. Geophys. Res., 89, 7541, 1984.

Davis, Jr., L. and Smith, E. J.: A model of Saturn's magnetic field based on all available data, J. Geophys. Res., 95, 15 257, 1990.

Edwards, T. M., Bunce, E. J., and Cowley, S. W. H.: A note on the vector potential of Connerney et al.'s model of the equatorial current sheet in Jupiter's magnetosphere, Planet. Space Sci., 49, 1115, 2001.

Espinosa, S. A. and Dougherty, M. K.: Periodic perturbations in Saturn's magnetic field, Geophys. Res. Lett., 27, 2785, 2000.

Giampieri, G. and Dougherty, M. K.: Modelling of the ring current in Saturn's magnetosphere, Geophys. Res. Lett., submitted, 2002.

Frank, L. A., Burek, B. G., Ackerson, K. L., Wolfe, J. H., and Mihalov, J. D.: Plasmas in Saturn's magnetosphere, J. Geophys. Res., 85, 5695, 1980.

Ness, N. F., Acuña, M. H., Lepping, R. P., Connerney, J. E. P., Behannon, K. W., Burlaga, L. F., and Neubauer, F.: Magnetic field studies by Voyager 1: Preliminary results at Saturn, Science, 212, 211, 1981.

Ness, N. F., Acuña, M. H., Behannon, K. W., Burlaga, L. F., Connerney, J. E. P., Lepping, R. P., and Neubauer, F.: Magnetic field studies by Voyager 2: Preliminary results at Saturn, Science, 215, 558, 1982.

Richardson, J. D.: Thermal ions at Saturn: Plasma parameters and implications, J. Geophys. Res., 91, 1381, 1986.

Richardson, J. D.: An extended plasma model for Saturn, Geophys. Res. Lett., 22, 1177, 1995.

Sittler, Jr., E. C., Ogilvie, K. W., and Scudder, J. D.: Survey of lowenergy plasma electrons in Saturn's magnetosphere: Voyagers 1 and 2, J. Geophys. Res., 88, 8848, 1983.

Smith, E. J., Davis, Jr., L., Jones, D., Coleman, Jr., P. J., Colburn, D. S., Dyal, P., and Sonett, C. P.: Saturn's magnetic field and magnetosphere, Science, 207, 407, 1980a.

Smith, E. J., Davis, Jr., L., Jones, D. E., Coleman, Jr., P. J., Colburn, D. S., Dyal, P., and Sonett, C. P.: Saturn's magnetosphere and its interaction with the solar wind, J. Geophys. Res., 85, 5655, $1980 b$. 